\title{
A Low-Cost Writing Lab Software for Teaching English as a Second Language
}

\author{
Han J. W. van Triest ${ }^{1,2}$, L Paul Mantha ${ }^{1 *}$, Yaonan Zhang ${ }^{1,2}$, Yan Kang ${ }^{1,2}$ \\ ${ }^{1}$ Sino-Dutch School of Biomedical and Information Engineering, \\ Northeastern University, Shenyang, China \\ ${ }^{2}$ Key Laboratory of Medical Image Computing, Ministry of Education, \\ Northeastern University, Shenyang, China \\ *Corresponding Author: paul@bmie.neu.edu.cn
}

\begin{abstract}
English Writing classes are very common at universities around the world, but are often considered cumbersome to teach. Moreover, the need for a fully equipped computer lab maybe problematic, and reduces the opportunity for students to collaborate. This paper examines how The Low Cost Writing Lab Software (Lo$c L a b)$ can be introduced into an English Writing class and how it has the ability to transform each traditional classroom into an advanced writing lab; one that is more student focused and increases the amount and variety of student collaboration during the process of writing an essay.
\end{abstract}

Keywords: Writing Labs; Language Teaching; Multi-Media Classroom

\section{Introduction}

English Language writing courses are taught to large numbers of students at universities in virtually every major. Some of these courses use a textbook that provides an exemplar for students to emulate [1] while others use texts that students have created while completing a task [2]. Both of these approaches could benefit from the use of a Computerized Learning Lab which allows the students and the instructor to interact with the text. [3] However, these Labs are of limited availability due to the cost of creating and maintaining them.

In addition from the point of view of collaborative learning, such Labs have a major drawback, i.e. they physically isolate the students in cubicles thus effectively limiting their ability to communicate with other students the one on either side of them. The other kind of classroom and possibly the most common is the multimedia room with a screen and projector. In this type of classrooms the instructor creates a presentation using a slideshow program like Microsoft PowerPoint and then comments on it, sometimes they ask the students to comment as well.[4]

One of the drawbacks of using $\mathrm{Mi}$ crosoft PowerPoint type software is that they do not allow for easy sharing during class and that they are allow difficult to edit during class, thus the instructor must think of the points that they wish to discuss and highlight them before class. Thus, the lesson is set before class begins and the instructor may attempt to elicit answers to predetermined questions which severely limits or eliminates peer review and other collaborative elements from the classroom part of the course.[4]

To overcome these problems, a collaborative writing tool, named LocLab, has been developed in house that has the ability to convert any class-room into an advanced writing lab. The software is light- 
weight, encourages collaborative learning, and is very intuitive and interactive in its nature enabling the instructor to emphasize any learning point that may come up during class.

\section{Materials and Methods}

The software LocLab is written in $\mathrm{C}++$, and based on the $Q t$ framework (Nokia, http://qt.nokia.com). It consists of two components, namely a server that is to be run on the instructor's computer, and a client component that runs on the students' computer. Currently, the LocLab is only available for Microsoft Windows, but can be converted for other platforms with minimal effort, due to the crossplatform nature of $Q t$. The software requires a minimal local network, preferably wireless. Below each of the components will be briefly described.

\subsection{Prerequisites}

In order to deploy LocLab in an educational environment, a few prerequisites need to be met. The class room should ideally be equipped with a digital projector and screen. The projector is used for the plenary discussion of students' work. Moreover, a local wireless network is required. In the present work, a mini USB-powered wireless router is employed, to set up this local network. The last prerequisite is a number of laptops brought by the students themselves. One can require for each student to bring his/her own laptop, but one laptop per (small) group is suitable as well, as it encourages collaborative writing.

\subsection{LocLab Server}

The server part of LocLab (see Fig. 1) functions as the center of all communication and will be used in lesson-planning, recording of student achievements and the reviewing of current student work.
The server contains a number of subcomponents, some of which will be discussed below.

- Text editor: The Server window prominently features a Rich Text Editor in which the instructor can review the current work by the students. The feedback created during the review will then be broadcasted either to the individual student, or to all students at the same time.

- Lesson Plan Creator: Before each class, the instructor can select from a list of previously designed student tasks for rapid lesson planning. The lessons will be loaded and the instructor can decide when to distribute these lessons to the students.

- HTTP Server: A HTTP server is included for the easy distribution of the client software amongst the students as well as distribution of other course ware.

- Homing Beacon: Once a student starts the client software, it will automatically try to connect to the server. This is achieved by setting up a homing beacon which will periodically send a blip using UDP multicast technology. Once detected by the client, it will automatically connect to the source IP-address.

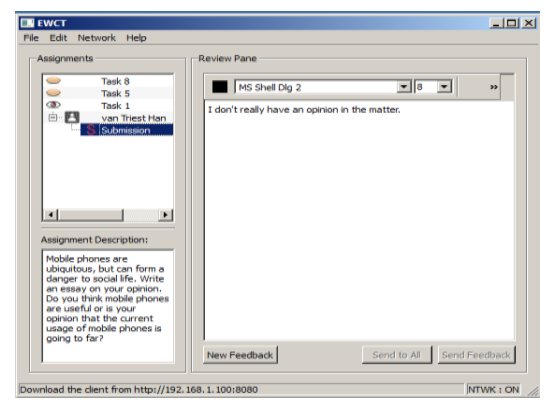

Fig 1: The LocLab Server component. 


\subsection{LocLab Client}

The second component of the LocLab framework is given by the writing class client. The purpose of the client is for students to rapidly submit their compositions and receive feedback from the instructor. It can be downloaded from the HTTP server incorporated in the server software, and for the most part consists of a Rich Text Editor. Other dominant features are the task selector in which the student can select which task he is to work on, and the task description widget, which describes the currently selected task. Students are to include their personal information before starting the software for the first time, and this information will be stored on the local computer as well as in the database running on the server. The client software can be seen in Figure 2.

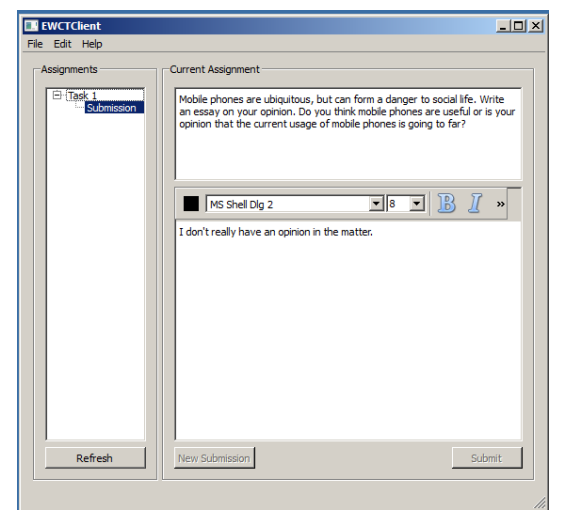

Figure 2: LocLab Client software

\section{Experiments and Results}

In order to field test The Low Cost Writing Lab Software (LocLab), it was employed in the latter part of two freshman English Writing classes. These classes were taught in a standard multimedia classroom equipped with a projector and screen. The classes were structured so that students worked collaboratively outside of class hours to produce either a part of a five paragraph essay: an introductory, body or concluding paragraph or a whole essay with topics chosen that would allow students to express their own ideas and create authentic texts to work from. In both before and after the introduction of LocLab the students would work collaboratively in groups.

Before the testing of LocLab, in the second half of the class a brainstorming session about the topic for the next week would take place and any remaining time was devoted to the students beginning work on the assignment. The students would email the assignment to the instructor who would then choose points in the text to comment on, create a PowerPoint with said text and present it to the students at the beginning of the next class. A largely one way discussion would ensure.

In the latter part, after introduction of Loclab, the students would still finish the assignment outside class, but the in-class structure changed. At the beginning of class the instructor using LocLab send the students a document, with the topic of the next writing, after which a brief brainstorming session was conducted and the students wrote a five sentence introduction to the topic which they then send back to the instructor who used the rely to all function thus allowing the students to have the paragraph to be commented on in front of them. They were then asked how they could make it better. The focus of the student groups moved from the projector screen at the front, to the laptop screen in the middle of the group. The instructor then use LocLab in a similar fashion to PowerPoint to allow whole class viewing. Moving from group to group the instructor asked for their comments and or corrections. The instructor could add their own comments during this process or wait until all the groups 
had finished and then add some of their own. Each of the groups writing were reviewed and commented on. The groups were then given the task of finishing the essay and emailing to the instructor for an asynchronous review.

\section{Discussion}

As demonstrated, LocLab has large potential in transforming writing classes into an immersive collaborative multimedia experience. The wireless network largely removes the cumbersome process of retrieving student work and demonstrating it to the students present in the class. Furthermore feedback can be sent to the students without effort, increasing the impact of writing classes, as students can see many different styles of writing from their peers.

The client software, however, currently only runs on Microsoft Windows. Moreover, students are to bring their own laptops, which can be cumbersome, or students may not be able to afford a laptop. Mobile platforms on the other hand, become increasingly powerful and many modern mobile phones are equipped with wireless capabilities. In the future the client will be extended to run on other platforms than Microsoft Windows, including android and $i O S$. As the platform has been written using $Q t$, a crossplatform Graphical User Interface (GUI) toolkit, only minor efforts are required.

\section{Conclusion}

In this paper The Low Cost Writing Lab Software (LocLab) was shown to be able to change the focus of the classroom, thus creating a more student centered environment. It allows students greater range of commenting and changes the driver of comments from the instructor to the students. LocLab also increases the amount of student collaboration from that of cre- ating a whole piece of writing to one an interconnected string of draft paragraph, peer-review and incorporation of comments to create a whole piece of writing. In addition it was shown that LocLab is easy to install and use.

\section{Acknowledgments}

This work has been partly sponsored by the Digital Image Processing Project by the Graduate Student Education program of Northeastern University, Shenyang, China.

\section{References}

[1] Qin Shou Sheng, Yang Tong Fu, Li Xiao Hong, Higher Education Press 2010

[2] Joseph Harris Teaching With Student Texts Utah State University Press 2010

[3] Daniel O. Jackson "Convergent and Divergent Computer-Mediated Communication Tasks in an English for Academic Purposes Course" The Electronic Journal for English as a Second Language. December 2011Volume 15, Number 3

[4] Lin Siew Fong "Benifits of Collaborative Writing for ESL Advanced Diploma Students in the Production of Reports" US-China Education Review B 42012 